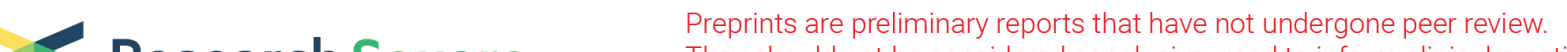 $\begin{array}{ll}\text { Research Square } & \text { They should not be considered conclusive, used to inform clinical practice, } \\ \text { or referenced by the media as validated information. }\end{array}$
}

\section{The prognosis of type III biliary atresia with a hilar cyst on ultrasonography might not be better than that without}

\section{Quan-yuan Shan}

Sun Yat-sen University First Affiliated Hospital

\section{Bao-xian Liu}

Sun Yat-sen University First Affiliated Hospital

\section{Zhi-hai Zhong}

Sun Yat-sen University First Affiliated Hospital

\section{Hua-dong Chen}

Sun Yat-sen University First Affiliated Hospital

\section{Yu Guo}

Sun Yat-sen University First Affiliated Hospital

\section{Xiao-yan Xie}

Sun Yat-sen University First Affiliated Hospital

\section{Wen-ying Zhou}

Sun Yat-sen University First Affiliated Hospital

\section{Hong Jiang}

Sun Yat-sen University First Affiliated Hospital

Lu-yao Zhou ( $\sim$ zhouly6@mail.sysu.edu.cn )

Sun Yat-sen University First Affiliated Hospital https://orcid.org/0000-0002-1828-0878

\section{Research article}

Keywords: Biliary atresia; cyst; ultrasonography; prognosis

Posted Date: July 1st, 2019

DOl: https://doi.org/10.21203/rs.2.10787/v1

License: (c) (i) This work is licensed under a Creative Commons Attribution 4.0 International License. Read Full License 


\section{Abstract}

Background Biliary atresia (BA) with a hilar cyst is an uncommon variant of BA that may be misunderstood to have a relatively favorable prognosis. Methods A single-center retrospective review of patients that matched BA with a hilar cyst $(n=27)$ with $B A$ without a cyst $(n=27)$ over a 5 -years period was done. All patients were diagnosed as type III BA by histologic examination and cholangiograms. Results There were no significant intergroup differences between baseline characteristics and outcomes after Kasai portoenterostomy surgery in two groups. The Kaplan-Meier survival curves showed no significant difference in cumulative native liver survival rate of the two groups $(p=0.584)$. Of the 27 BA patients with a hilar cyst, 8 were showed the cyst communicated with the gallbladder on cholangiograms, and KaplanMeier survival curves indicated that the cumulative native liver survival rate was significantly shorter $(P=0.045)$ in them than those who the cyst was not communicated with the gallbladder. Conclusions Type III BA with a hilar cyst had no better prognosis compared with Type III BA without a cyst. A cyst communicated with the gallbladder may be associated with a poor eventual outcome for Type III BA with a hilar cyst.

\section{Background}

Biliary atresia (BA) is a destructive, inflammatory, obliterative cholangiopathy that develops in $1 / 5,000$ to $1 / 19,000$ newborns [1]. BA affects varying lengths of both the extra and intrahepatic biliary tract and is usually classified, according to the most proximal level of obstruction, into 3 types. Type I BA is atresia of the common bile duct (CBD), which is often cystic. Type II BA is atresia of the hepatic duct and can contain visible patent ducts at porta. Type III BA $(>90 \%)$ is atresia at the porta hepatis, and only microscopic ductules can be detected $[1 ; 2]$.

In $6 \%$ to $20 \%$ of BA cases [3-5], a cyst or cysts, which usually could be clearly visualized on Ultrasound scan $[6,7]$, are presented at diagnosis in any part of the extrahepatic biliary tract. Some of these cases belong to Type I BA, that is, so called cystic BA. Some other of these cases belongs to Type III BA. Nevertheless, some researchers suggest that BA with a hilar cyst is a clinically distinct subtype associated with projected improved outcomes [8, 9], while other researchers have not found this [10].

To clarify this issue, we retrospectively reviewed the characteristics of preoperative ultrasonography (USG) findings and the prognosis of 27 BA patients who had a hilar cyst and accepted Kasai portoenterostomy (KPE) surgery, and compared them with a matched pair control group of 27 BA patients without a hilar cyst.

\section{Methods}

\section{Study population}

The First Affiliated Hospital, Sun Yat-sen University, Guangzhou, China, has a large tertiary center of pediatric hepatobiliary disease with referrals largely from the southeast of China. We retrospectively 
identified all infants who was diagnosed as BA with a hilar cyst (group A) by USG during April 2012 to June 2018. This study was approval from our institutional review board, and we obtained written informed consent from the parents or guardians of the children.

The inclusion criteria for the study were 1) patients were identified to have a cyst or cysts at porta on preoperative USG; 2) the diagnosis of BA was confirmed in all cases by histologic examination of resected biliary remnants and cholangiograms; 3 ) all patients were treated by KPE; 4) the follow up period was more than 6 months unless the patient death before 6 months.

As all patients in group A were diagnosed as type III BA by histologic examination of resected biliary remnants and cholangiograms finally, a group of infants with isolated type III BA without cyst (group B) was used for comparison. All group B patients were also treated by KPE during the same period and had no cysts at porta on preoperative USG. The control patients of group B were matched in a 1:1 fashion based on gender and age. And the gender of group $B$ patients was totally the same with group $A$, while the difference of age at diagnosis between matched-pair patients in group $B$ and patients in group $A$ was no more than 4 days.

The exclusion criteria for the study were 1) the pathological result was unclear; 2) patients had not undergone KPE; 3) patients had not undergone USG within one month before KPE; 4) patients had extrahepatic anomalies.

\section{Ultrasonography technique and imaging analysis}

Ultrasound examinations were all performed by one experienced pediatric radiologist (ZLY, 9 years of experience in hepatobiliary imaging). For the purposes of the study, images and reports were retrospectively reviewed.

All examinations were performed after 2-6 hours of fasting. B-mode ultrasound and color Doppler were used with an AixPlorer scanner (Supersonic Imagine, Paris, France) incorporating a linear-array transducer (SL15-4) and a curved-array transducer (SC6-1). We recoded information about USG, such as the number of cysts, diameter of the biggest cyst, and whether the cyst was communicated with the gallbladder. When an anechoic tube was found between the cyst and the gallbladder on USG, it was recorded as the cyst communicated with the gallbladder. When no tube was found between the cyst and the gallbladder on USG, it was recorded as the cyst not communicated with the gallbladder. For patients with large gallbladder (longer than $1.5 \mathrm{~cm}, \mathrm{n}=16$ ), whether the cyst communicated with the gallbladder was confirmed by intraoperative cholangiography. For patients with small gallbladder (shorter than 1.5 $\mathrm{cm}, \mathrm{n}=6)$ and absence of gallbladder on USG $(\mathrm{n}=5)$, whether the cyst communicated with the gallbladder wasn't confirmed by intraoperative cholangiography, because it is generally impossible to perform intraoperative cholangiography in these patients. In addition, two patients with cyst communicated with the gallbladder were confirmed by the percutaneous ultrasound-guided cholecystocholangiography with microbubbles, a mature technology in our center [11]. 
Figure 1 shows a 9-day-old female with a $28 \mathrm{~mm} \times 16 \mathrm{~mm}$ porta cyst that was communicated with the gallbladder from a small tube (approximately $2 \mathrm{~mm}$ diameter) (Fig. 1a). And the result was confirmed by the percutaneous ultrasound-guided cholecystocholangiography with microbubbles (Fig. 1b).

Figure 2 shows a 30-day-old male with a $7.0 \mathrm{~mm} \times 5.0 \mathrm{~mm}$ porta cyst that was not communicated with the gallbladder.

Liver stiffness measurement was performed by real-time shear-wave elastography (SWE). The infants were kept quiet by feeding while liver stiffness measurements were taken. Segment V or VI was selected as the target area for measurement. All SWE examinations were performed by a single sonographer (L.Y.Z., 3 years of experience with elastography) with an epigastric transducer position. The transducer was kept perpendicular to the skin, and no pressure was given during the SWE examination. SWE was performed in dual mode (i.e., elastograms displayed alongside gray-scale sonograms in real time). We have detailed our SWE examination protocol previously [12]. The average values of the three readings in each infant were used for subsequent statistical analyses.

\section{Data collection}

Follow-up continued until liver transplant, death, withdrawal from the study, or January 1, 2019. The postoperative course in terms of bile flow, achievement of a jaundice-free state and postoperative cholangitis in each group was studied. Jaundice free was defined as a total serum bilirubin of $<2.0 \mathrm{mg} / \mathrm{dl}$ at 6 months following surgery [13].

Statistical parameters obtained from our hospital included age at diagnosis, sex, and laboratory values, including conjugated hyperbilirubinemia, serum gamma-glutamyltransferase and serum cholesterol.

\section{Statistical analysis}

Descriptive data are summarized as the mean \pm standard deviation (SD) for continuous variables and as percentages for categorical variables. Statistical analysis was performed using the chi-squared test or Student's t test, as appropriate. The differences in survival with native liver among groups were analyzed by the Kaplan-Meier Method with endpoints of death or liver transplantation and compared by using the log-rank test. A probability $(P)$ value of $<0.05$ was considered statistically significant. Data were analyzed with SPSS, version 14 for Windows (SPSS, Inc., Chicago, IL).

\section{Results}

Of 326 infants with BA, 30 (9.2\%) had BA with a cyst, including 14 males. Three patients ( 1 male and 2 females) in 30 were lost to follow-up 3 months after KPE. At last, there were 27 patients in each group, with 13 males and 14 females. The mean patient age at diagnosis was $54.3 \pm 19.8$ days (range 9-96 days). 


\section{Comparisons of patient characteristics and outcomes between BA patients with hilar cyst and BA patients without a hilar cyst}

After matching, there were no significant differences between group $A$ and group $B$ in age, sex, laboratory values [total bilirubin (TB), direct bilirubin (DB), isolated bilirubin (IB), alanine transaminase (ALT), and aspartate transaminase (AST)], follow-up period or the interval between diagnosis and surgery (days) (Table 1). Bile flow appeared in 23/27 (85.2\%) of the group A and $25 / 27(92.6 \%)$ of group B $(p=0.39)$. A jaundice-free state was achieved in 15/27 (55.6\%) and 17/27 (63.0\%) patients in group A and group B, respectively $(p=0.58)$. The postoperative cholangitis rate was similar in two groups: $11 / 27(40.78 \%)$ v.s. 7/27 (24.8\%), $p=0.25$ Transplant rate was also similar: 9/27 (33.3\%) and 12/27 (44.4\%) in group $A$ and group $B$, respectively $(p=0.40)$. The mean time from surgery until transplant was $7.7 \pm 1.9$ months (range 5-11 months) in group $A$ and $10.6 \pm 4.6$ months (range 4-20 months) in group $B(p=0.09)$. The KaplanMeier native liver survival curves (Fig. 3 ) showed no significant difference in cumulative native liver survival rate between two groups $(p=0.58)$.

\section{USG findings of BA patients with hilar cyst}

The mean diameter of the cyst, assessed at preoperative ultrasound, was 7.1 $\pm 5.0 \mathrm{~mm}$ (range 3.0-28.0 $\mathrm{mm}$ ). There was one patient with multiple cysts, and the other 26 were solitary. There were eight patients with a solitary cyst communicated with the gallbladder. The other 19 patients' cysts were not communicated with the gallbladder. The SWE value at the time of diagnosis was $12.8 \pm 3.7 \mathrm{kPa}$ in the group $A$ and $13.9 \pm 6.4 \mathrm{kPa}$ in the group $B(p=0.47)$. There were no significant differences between group $A$ and group $B$ in all USG findings, including absence of gallbladder rate, length and width of gallbladder, portal vein diameter, diameters of the proximal right hepatic artery, oblique diameter of the right liver lobe, rough echo of liver parenchyma rate, and spleen diameter.

\section{Comparisons of patient characteristics, outcomes and USG findings between patients with the cyst} communicated with the gallbladder and patients with hilar cyst not communicated with the gallbladder

The eight patientswith the cyst communicated with the gallbladder (group C) were compared to the other nineteen patients with the cyst not-communicated with the gallbladder (group D) (Table 2). There were no significant differences between the two groups in baseline characteristics at KPE surgery. After KPE surgery, a jaundice-free state was achieved in $2 / 8$ (25.0\%) and 13/19 (68.4\%) patients in group $C$ and group $D$, respectively $(p=0.04)$. And the post-operative death rate was significant higher in the group $C$ than in the group $D(4 / 8(50.0 \%)$ v.s. $1 / 19(5.3 \%), p=0.01)$. The other outcomes were similar in group $C$ and group D. The Kaplan-Meier native liver survival curves (Fig. 4) showed better cumulative native liver survival rate in the group $D$ patients $(p=0.045)$.

There were no significant differences between group $C$ and group D in USG characteristics, apart from length of gallbladder ( $32.3 \pm 8.7 \mathrm{~mm}$ in group $C$ v.s. $19.0 \pm 12.1 \mathrm{~mm}$ in group $D, p=0.01)$, and spleen diameter ( $54.5 \pm 9.4 \mathrm{~mm}$ in group $C$ v.s. $63.8 \pm 9.2 \mathrm{~mm}$ in group $\mathrm{D}, \mathrm{p}=0.03)$. 


\section{Discussion}

This matched-pair, retrospective cohort study revealed that the outcome of type III BA with hilar cyst revealed on USG was no better than isolated type III BA without cyst. Our results further indicated that in type III BA with a hilar cyst, the outcomes of KPE might be worse when the hilar cyst was found to have a communication with the gallbladder. This finding was very important because it reminded that the pediatric surgeons should take type III BA patients that had hilar cyst as careful as those who had not, especially when the hilar cyst was communicated with the gallbladder.

The incidence of extrahepatic cysts in BA ranges from $6 \%$ to $20 \%$ [3-5], which is in accordance with the current study $(30 / 326,9.2 \%)$. Several studies $[5 ; 14 ; 15]$ had mentioned that BA with hilar cyst was a clinically distinct subtype associated with better prognosis, which was different from our study. The difference might be caused by the diversity of population composition. In their studies, there included many patients with cystic BA (Type I), which was widely considered as correctable BA with favorable outcomes. In Caponcelli et al.'s study [8], $41 \%$ of cystic BA infants were diagnosed by antenatal ultrasonography and, particularly, with early surgery. They found that age at KPE markedly affected outcome, which may be another true reason for the better prognosis in patients with cystic BA.

In our study, all BA patients with cyst were diagnosed as type III BA by histologic examination of resected biliary remnants and cholangiograms. And we compared their outcomes with a matched pair control group of isolated type III BA patients without cyst. After matching, the baseline characteristics at KPE surgery, including age, gender and liver function, were similar between two groups. Thus, it was reasonable that our study showed the prognosis of BA with hilar cyst was not better than that without. Some other researchers $[9 ; 10]$ reported similar outcomes, especially in type III BA.

The current study showed that outcomes after KPE surgery, such as bile flow rate, jaundice-free state rate, postoperative cholangitis rate, transplant rate, post-operative death rate, and time from surgery until transplant, were not significantly different between patients with hilar cyst and patients without hilar cyst. Kaplan-Meier survival curves indicated that the overall survival time with a native liver after KPE between two groups was also not significantly different. SWE value, which is widely used to measure liver stiffness and has the possibility for predicting native liver survival after KPE surgery in patients with isolated BA $[11,16,17]$, was also similar between two groups. And our analyses revealed that most USG characteristics were similar between two groups. It suggested that similar pathological changes might exist on the two groups, which may be the underlying cause of no better prognosis in BA patients with hilar cyst.

As Kaplan-Meier survival curves indicated that the overall survival time with a native liver after KPE was significantly longer in BA patients with the cyst not-communicated with the gallbladder than BA patients with the cyst communicated with the gallbladder in our study, we then compared their clinical characteristics. We found out that most USG characteristics about liver were similar between two groups, and liver function before KPE was also similar. What's more, the SWE value was also similar between two groups. And all the above signs indicated that liver pathological changes were similar between two 
groups. We conjectured that a cyst communicated with the gallbladder on USG might be associated with more severe malformation of the biliary system than a cyst not communicated with the gallbladder, which might be one possible reason for the poorer outcome.

We admit several limitations to our study. First, the conclusions have been inadequately persuasive due to the insufficient number of cases and retrospective design of the study. Second, we did not further classify the type III BA with hilar cyst, which might imply the real reason for different prognosis between patients with a cyst communicated with the gallbladder on USG and patients with a cyst not communicated with the gallbladder. What's more, we did not correlate SWE results from pathological fibrosis grades. Despite those limitations, this remains the largest study of BA patients with hilar cyst.

\section{Conclusions}

In conclusion, type III BA with a hilar cyst had no better prognosis compared with Type III BA without a cyst. In addition, for Type III BA with a hilar cyst, the cyst communicated with the gallbladder may be associated with a poor eventual outcome.

\section{Abbreviations}

Biliary atresia, BA

Common bile duct, CBD

Ultrasonography, USG

Kasai portoenterostomy, KPE

Shearwave elastography, SWE

Standard deviation, SD

Probability, $\mathrm{P}$

Total bilirubin, TB

Direct bilirubin, DB

Isolated bilirubin, IB

Alanine transaminase, ALT

Aspartate transaminase, AST

\section{References}


Hartley JL, Davenport M, Kelly DA. Biliary atresia. Lancet 2009;374:1704-13

2 Superina R, Magee JC, Brandt ML, et al. The anatomic pattern of biliary atresia identified at time of Kasai hepatoportoenterostomy and early postoperative clearance of jaundice are significant predictors of transplant-free survival. Ann Surg 2011;254:577-85

3 Arima E, Fonkalsrud EW, Neerhout RC. Experience in the management of surgically correctable biliary atresia. Surgery 1974;75:228-32

4 Lilly JR, Hall RJ, Vasquez-Estevez J, et al. The surgery of "correctable" biliary atresia. J Pediatric Surg 1987;22:522-5

5 Karrer FM, Lilly JR, Stewart BA, et al. Biliary atresia registry, 1976 to 1989. J Pediatric Surg 1990;25:1076-80; discussion 1081

6 Koob M, Pariente D, Habes D, et al. The porta hepatis microcyst: an additional sonographic sign for the diagnosis of biliary atresia. Eur Radiol 2017;27:1812-21

7 Kim MJ, Park YN, Han SJ, et al. Biliary atresia in neonates and infants: triangular area of high signal intensity in the porta hepatis at T2-weighted MR cholangiography with US and histopathologic correlation. Radiology 2000;215:395-401

8 Caponcelli E, Knisely AS, Davenport M. Cystic biliary atresia: an etiologic and prognostic subgroup. J Pediatric Surg 2008;43:1619-24

9 Lal R, Prasad DK, Krishna P, et al. Biliary atresia with a "cyst at porta": management and outcome as per the cholangiographic anatomy. Pediatr Surg Int 2007;23:773-8

10 Hill SJ, Clifton MS, Derderian SC, et al. Cystic biliary atresia: a wolf in sheep's clothing. Am Surg 2013;79:870-2

11 Zhou LY, Chen SL, Chen HD, et al. Percutaneous US-guided Cholecystocholangiography with Microbubbles for Assessment of Infants with US Findings Equivocal for Biliary Atresia and Gallbladder Longer than $1.5 \mathrm{~cm}$ : A Pilot Study. Radiology 2018;286:1033-9

12 Chen S, Liao B, Zhong Z, et al. Supersonic shearwave elastography in the assessment of liver fibrosis for postoperative patients with biliary atresia. Sci Rep 2016;6:31057

13 Nio M, Ohi R, Miyano T, et al. Five- and 10-year survival rates after surgery for biliary atresia: a report from the Japanese Biliary Atresia Registry. J Pediatric Surg 2003;38:997-1000

14 Ohi R, Nio M, Chiba T, et al. [The present status and problems in the treatment of biliary atresia with special reference to surgical and long-term results]. Nihon Geka Gakkai Zasshi 1989;90:1339-42 
15 Deguchi E, Iwai N, Yanagihara J, et al. Relationship between intraoperative cholangiographic patterns and outcomes in biliary atresia. Eur J Pediatr Surg 1998;8:146-9

16 Shin NY, Kim MJ, Lee MJ, et al. Transient elastography and sonography for prediction of liver fibrosis in infants with biliary atresia. J Ultrasound Med 2014;33:853-64

$17 \mathrm{Wu}$ JF, Lee CS, Lin WH, et al. Transient elastography is useful in diagnosing biliary atresia and predicting prognosis after hepatoportoenterostomy. Hepatology 2018;68:616-24

\section{Tables}

Table 1. Patient characteristics and outcomes 


\begin{tabular}{|c|c|c|c|}
\hline & $\begin{array}{l}\text { Group A } \\
(\mathrm{n}=27)\end{array}$ & Group B $(\mathrm{n}=27)$ & $\mathrm{P}$ value \\
\hline \multicolumn{2}{|l|}{ Baseline characteristics at KPE surgery } & $13(48.1 \%)$ & 1.00 \\
\hline Age at surgery (days) & $65.9 \pm 13.9$ & $65.0 \pm 15.8$ & 0.74 \\
\hline Age at diagnosis (days) & $54.3 \pm 19.8$ & $54.5 \pm 19.7$ & 0.96 \\
\hline Diagnosis and surgical intervention (days) & $11.6 \pm 9.4$ & $10.6 \pm 11.1$ & 0.49 \\
\hline TB (umol/L) & $191.8 \pm 57.9$ & $185.9 \pm 70.4$ & 0.87 \\
\hline DB (umol/L) & $115.3 \pm 29.6$ & $113.1 \pm 44.7$ & 0.83 \\
\hline IB (umol/L) & $76.6 \pm 35.8$ & $79.0 \pm 36.4$ & 0.96 \\
\hline $\operatorname{ALT}(\mathrm{U} / \mathrm{L})$ & $150.0 \pm 84.6$ & $139.2 \pm 66.4$ & 0.86 \\
\hline AST (U/L) & $224.1 \pm 84.3$ & $241.7 \pm 160.6$ & 0.41 \\
\hline SWE value $₫ \mathrm{KPa} \square$ & $12.8 \pm 3.7$ & $13.9 \pm 6.4$ & 0.59 \\
\hline \multicolumn{4}{|l|}{ Outcomes after KPE surgery } \\
\hline Follow-up period $\square$ month $\square$ & $24.6 \pm 21.2$ & $35.9 \pm 21.4$ & 0.06 \\
\hline Bile flow (rate) & $23(85.2 \%)$ & $25(92.6 \%)$ & 0.39 \\
\hline A jaundice free state (rate) & $15(55.6 \%)$ & $17(63.0 \%)$ & 0.58 \\
\hline Post-operative cholangitis (rate) & $11(40.7 \%)$ & 7 (25.9\%) & 0.25 \\
\hline Transplant (rate) & $9(33.3 \%)$ & $12(44.4 \%)$ & 0.40 \\
\hline Time from surgery until transplant (months) & $7.7 \pm 1.9$ & $10.6 \pm 4.6$ & 0.11 \\
\hline Post-operative death (rate) & $5(18.5 \%)$ & $1(3.7 \%)$ & 0.08 \\
\hline \multicolumn{4}{|l|}{ USG characteristics } \\
\hline Absence of gallbladder (rate) & $5(18.5 \%)$ & $4(14.8 \%)$ & 0.72 \\
\hline Length of gallbladder (mm) & $24.3 \pm 13.74 .4 .6 \pm 4.1$ & $16.9 \pm 7.5$ & 0.11 \\
\hline Width of gallbladder (mm) & $0(0.00 \%)$ & $4.0 \pm 1.7$ & 0.59 \\
\hline TC sign negative (rate) & $4.1 \pm 0.8$ & $5(18.5 \%)$ & 0.051 \\
\hline Portal vein diameter (mm) & $2.0 \pm 0.4$ & $4.2 \pm 0.5$ & 0.85 \\
\hline Diameters of the proximal right hepatic artery $(\mathrm{mm})$ & $75.1 \pm 6.8$ & $2.0 \pm 0.5$ & 0.90 \\
\hline Oblique diameter of the right liver lobe (mm) & $6(22.2 \%)$ & $73.6 \pm 8.4$ & 0.40 \\
\hline Rough echo of liver parenchyma (rate) & $62.0 \pm 9.9$ & $5(18.5 \%)$ & 0.74 \\
\hline Spleen diameter (mm) & & $61.4 \pm 8.0$ & 0.82 \\
\hline
\end{tabular}

Group A, BA patients with cyst; and Group B, BA patients without cyst. KPE, Kasai portoenterostomy; TB, total bilirubin; DB, direct bilirubin; IB, isolated bilirubin; ALT, alanine transaminase; AST, aspartate transaminase; SWE, shearwave elastography; USG, ultrasonography; TC, triangular cord.

Table 2. Comparisons between the cyst communicated with the gallbladder or not in BA with a hilar cyst patients. 


\begin{tabular}{|c|c|c|c|}
\hline & Group C $(n=8)$ & Group D $(\mathrm{n}=19)$ & $\mathrm{P}$ value \\
\hline $\begin{array}{l}\text { Baseline characteristics at KPE surgery } \\
\text { Male (rate) }\end{array}$ & $4 / 8(50.0 \%)$ & $9 / 19(47.4 \%)$ & 0.90 \\
\hline Age at surgery (days) & $62.8 \pm 9.1$ & $67.3 \pm 15.9$ & 0.46 \\
\hline Age at diagnosis (days) & $45.9 \pm 19.5$ & $57.9 \pm 19.9$ & 0.16 \\
\hline Diagnosis and surgical intervention (days) & $16.9 \pm 11.7$ & $9.4 \pm 7.8$ & 0.06 \\
\hline TB (umol/L) & $190.4 \pm 70.2$ & $192.4 \pm 56.4$ & 0.94 \\
\hline $\mathrm{DB} \square \mathrm{umol} / \mathrm{L} \square$ & $113.4 \pm 33.9$ & $116.0 \pm 29.7$ & 0.85 \\
\hline IBロumol/L口 & $76.9 \pm 43.4$ & $76.4 \pm 34.9$ & 0.98 \\
\hline ALT (U/L) & $151.3 \pm 101.7$ & $149.4 \pm 82.9$ & 0.96 \\
\hline AST(U/L) & $228.1 \pm 41.3$ & $222.5 \pm 78.6$ & 0.89 \\
\hline SWE value (KPa) & $13.3 \pm 4.3$ & $12.6 \pm 3.6$ & 0.68 \\
\hline \multicolumn{4}{|l|}{ Outcomes after KPE surgery } \\
\hline Bile flow (rate) & $6 / 8(75.0 \%)$ & $17 / 19(89.5 \%)$ & 0.33 \\
\hline A jaundice free state (rate) & $2 / 8(25.0 \%)$ & $13 / 19(68.4 \%)$ & 0.04 \\
\hline Post-operative cholangitis (rate) & $1 / 8(12.5 \%)$ & $10 / 19(52.6 \%)$ & 0.053 \\
\hline Transplant (rate) & $2 / 8(25.0 \%)$ & $7 / 19(36.8 \%)$ & 0.55 \\
\hline Post-operative death (rate) & $4 / 8(50.0 \%)$ & $1 / 19(5.3 \%)$ & 0.01 \\
\hline \multicolumn{4}{|l|}{ USG characteristics } \\
\hline Cyst diameter (mm) & $10.0 \pm 7.9$ & $5.9 \pm 2.8$ & 0.052 \\
\hline Multiple cysts (rate) & $0 / 8(0.0 \%)$ & $1 / 19(5.3 \%)$ & 0.51 \\
\hline Absence of gallbladder (rate) & $0 / 8(0.0 \%)$ & $5 / 19(26.3 \%)$ & 0.11 \\
\hline Length of gallbladder (mm) & $32.3 \pm 8.7$ & $19.0 \pm 12.1$ & 0.01 \\
\hline Width of gallbladder (mm) & $4.7 \pm 2.7$ & $4.4 \pm 4.3$ & 0.86 \\
\hline Portal vein diameter (mm) & $4.5 \pm 0.9$ & $3.9 \pm 0.6$ & 0.051 \\
\hline Diameters of the proximal right hepatic artery (mm) & $2.0 \pm 0.5$ & $2.0 \pm 0.4$ & 0.95 \\
\hline Oblique diameter of the right liver lobe (mm) & $74.3 \pm 9.1$ & $75.1 \pm 5.9$ & 0.77 \\
\hline Rough echo of liver parenchyma (rate) & $1 / 8(12.5 \%)$ & $5 / 19(26.3 \%)$ & 0.43 \\
\hline Spleen diameter (mm) & $54.5 \pm 9.4$ & $63.8 \pm 9.2$ & 0.03 \\
\hline
\end{tabular}

Group C, BA with a hilar cyst patients with the cyst communicated with the gallbladder; and Group D, BA with a hilar cyst patients with the cyst not-communicated with the gallbladder. KPE, Kasai portoenterostomy; TB, total bilirubin; DB, direct bilirubin; IB, isolated bilirubin; ALT, alanine transaminase; AST, aspartate transaminase; SWE, shearwave elastography; USG, ultrasonography.

\section{Figures}




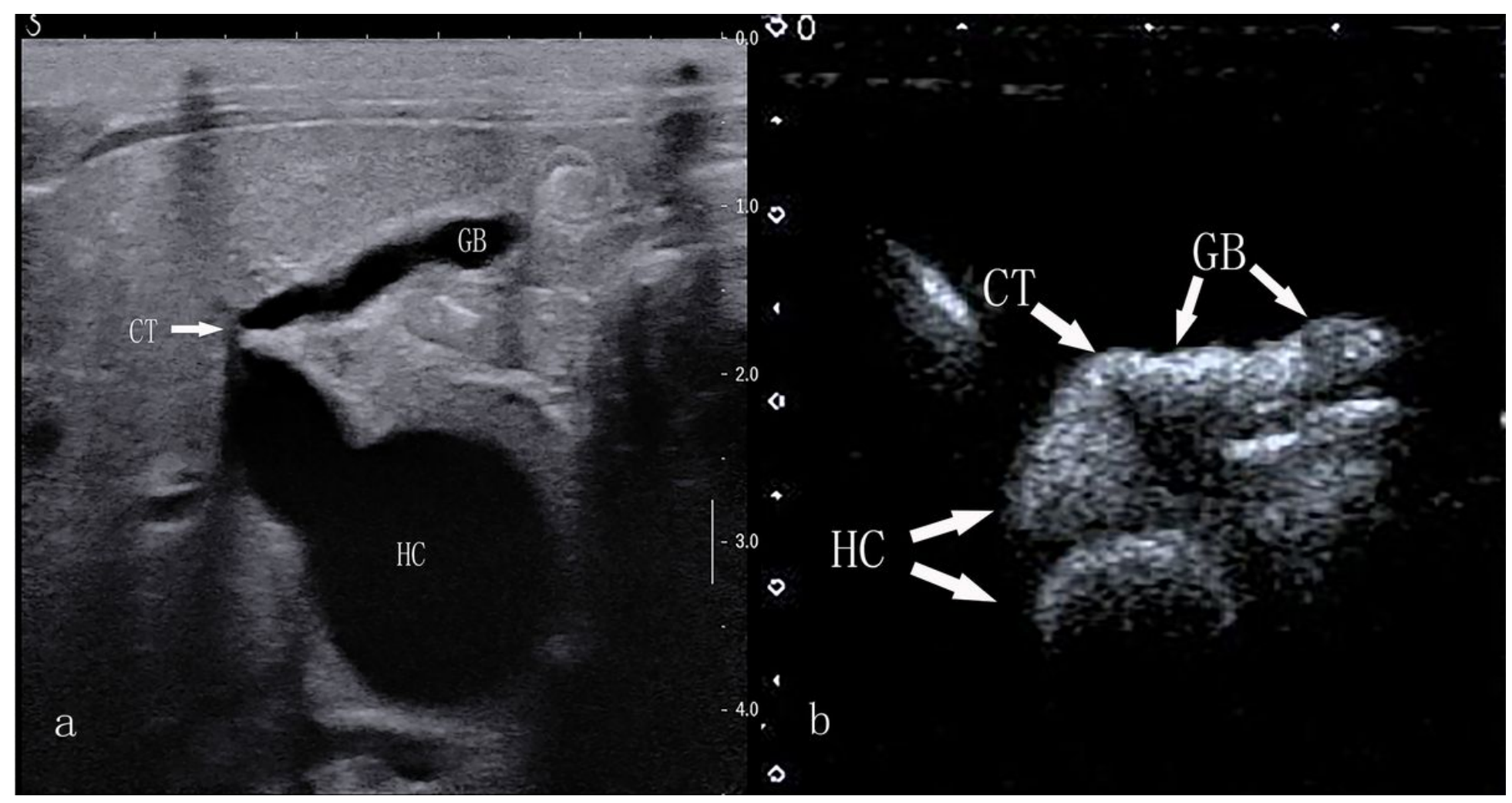

\section{Figure 1}

USG of a 9 days-old female with hilar cyst $(28 \mathrm{~mm} \times 16 \mathrm{~mm})$, which is communicated with gallbladder. (a) Gray-scale ultrasonography. (b) Contrast-enhanced ultrasonography obtained after injection of $5.0 \mathrm{~mL}$ of contrast material in the hilar cyst by the percutaneous ultrasound-guided cholecystocholangiography. Image obtained shows that hilar cyst, gallbladder and the communicating tube were all filled with contrast material. GB, gallbladder, $\mathrm{CT}$, communicating tube, and $\mathrm{HC}$, hilar cyst. 


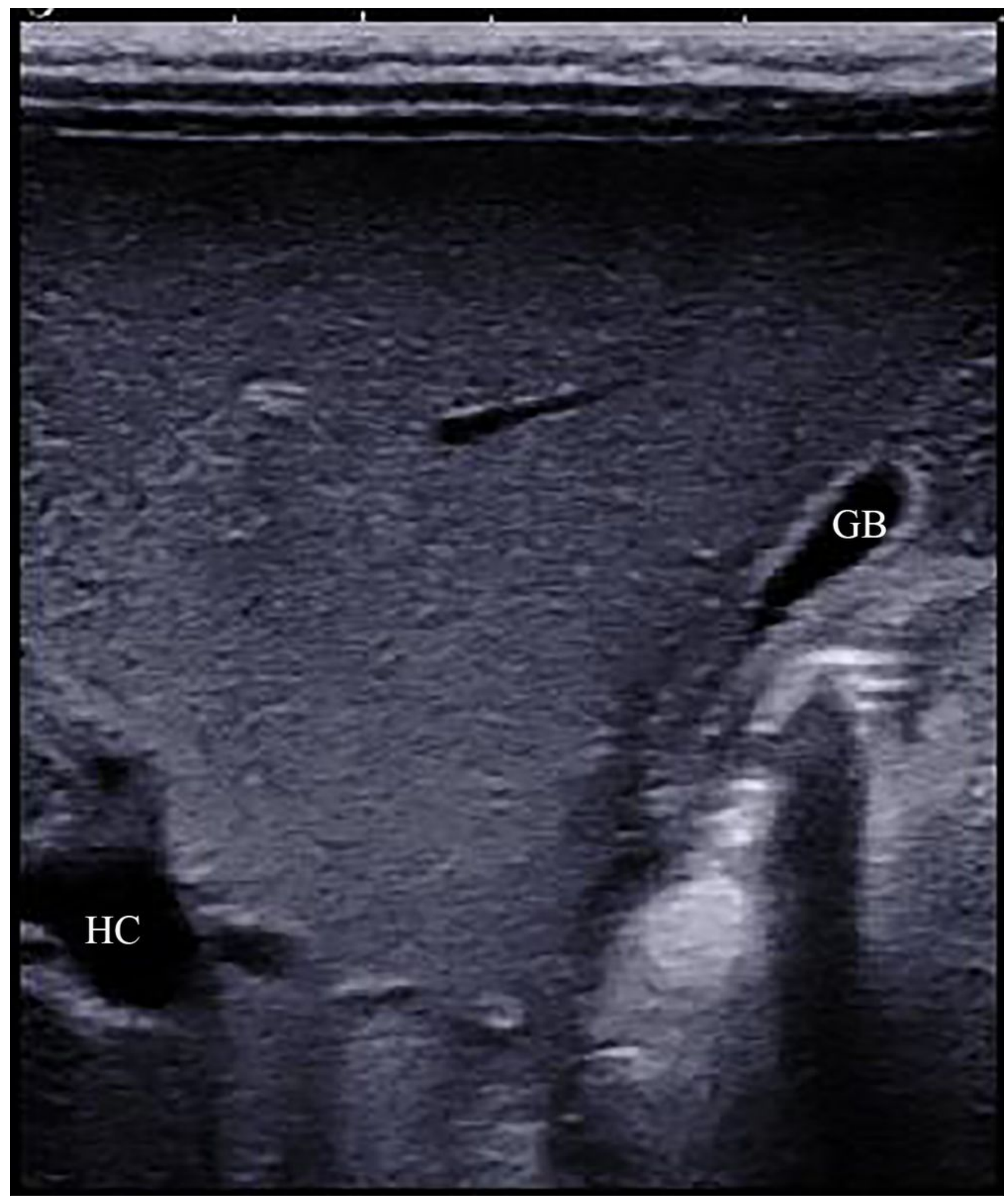

\section{Figure 2}

USG of a 30 days-old male with hilar cyst $(7.0 \mathrm{~mm} \times 5.0 \mathrm{~cm})$, which is not communicated with gallbladder. $\mathrm{GB}$, gallbladder, and $\mathrm{HC}$, hilar cyst. 


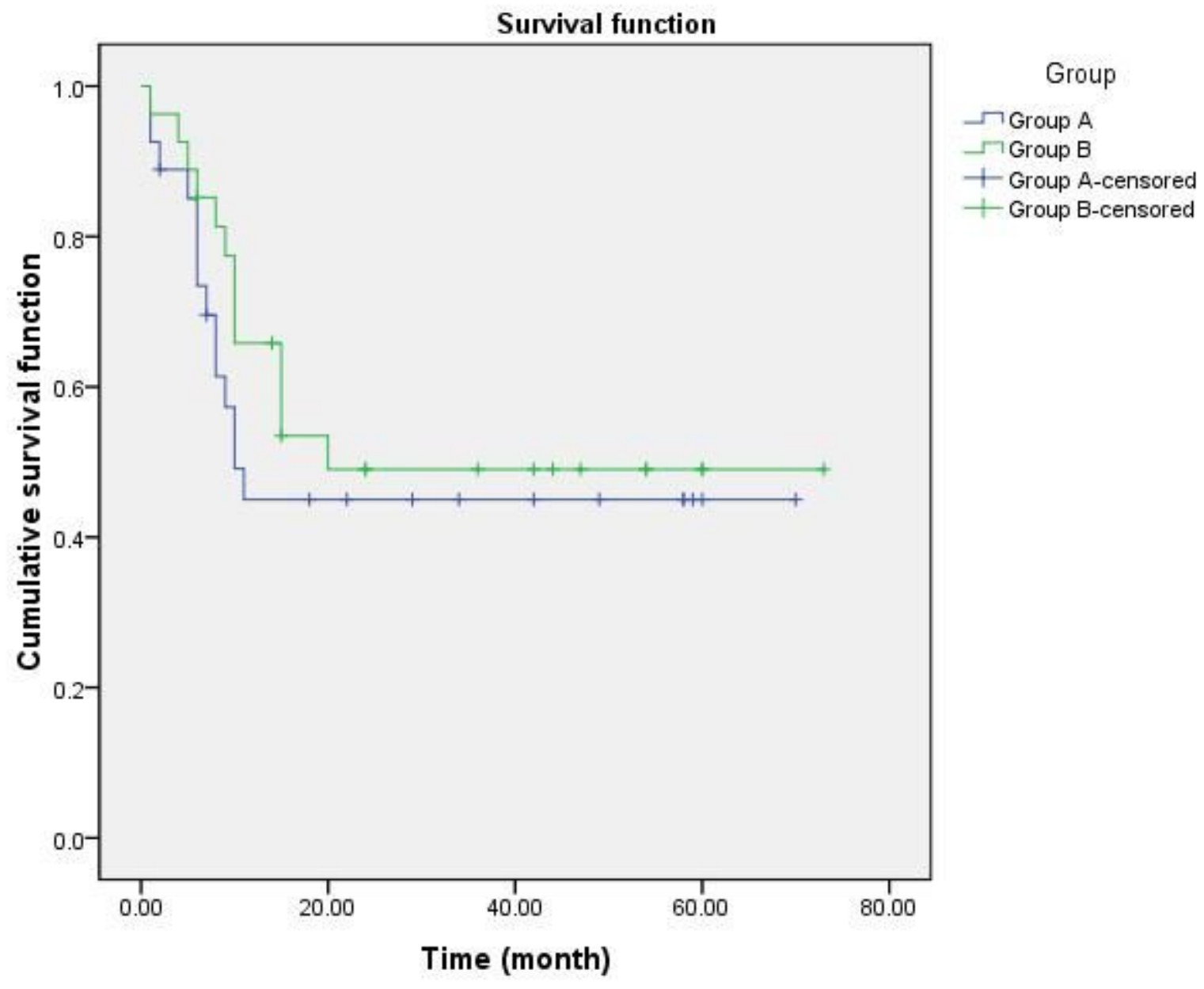

Figure 3

Kaplan-Meier native liver survival curves between group A and group $B$. There was no significant difference in cumulative native liver survival rate between group $A$ and group $B(p=0.58$, log-rank test). Group A, BA patients with cyst; and Group B, BA patients without cyst. 


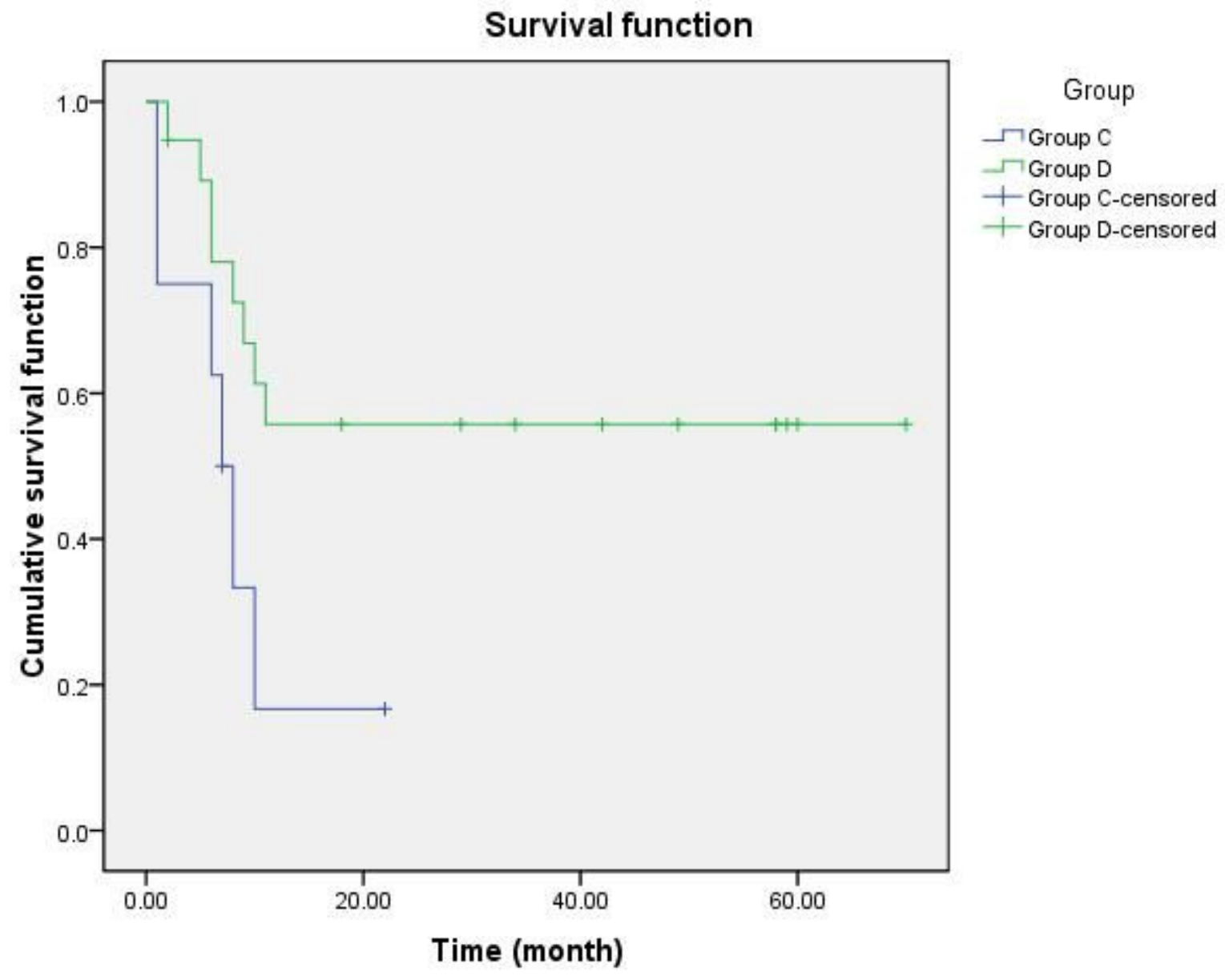

Figure 4

Kaplan-Meier native liver survival curves between group $C$ and group $D$. The cumulative native liver survival rate between group $C$ and group $D$ was stastically significant $(p=0.045$, log-rank test). Group $C$, BA with a hilar cyst patients with the cyst communicated with the gallbladder; and Group D, BA with a hilar cyst patients with the cyst not-communicated with the gallbladder. 